\title{
Leptomeningeal Carcinomatosis Secondary to Esophageal Cancer Diagnosed on Cytology
}

\author{
Sunil Vitthalrao Jagtap ${ }^{1}$ Shoaib Khoja ${ }^{1}$ Shubham \\ ${ }^{1}$ Department of Pathology, Krishna Institute of Medical Sciences \\ University, Karad, Maharashtra, India \\ ${ }^{2}$ GMC VMC Solapur, Maharashtra, India \\ ${ }^{3}$ Department of Oncology and Radiotherapy, Krishna Hospital and \\ Medical Research Centre, Karad, Maharashtra, India \\ ${ }^{4}$ Department of Surgery, Krishna Hospital and Medical Research \\ Centre, Karad, Maharashtra, India
}

\author{
S. Jagtap ${ }^{2}$ Rashmi Gudur ${ }^{3}$ Hemant Janugade ${ }^{4}$
}

\author{
Address for correspondence Sunil Vitthalrao Jagtap, MD, \\ Department of Pathology, Krishna Institute of Medical Sciences, \\ University, Karad, Maharashtra, India (e-mail: drsvjagtap@gmail.com).
}

\author{
Abstract \\ Keywords \\ - esophageal cancer \\ - neoplastic meningitis \\ - leptomeninges
}

Esophageal carcinoma is considered a common malignancy across the globe. These patients usually present with an advanced stage of the disease at the time of diagnosis. Herein, we report a 55-year male patient who presented with leptomeningeal carcinomatosis, detected on cerebrospinal fluid (CSF) cytology in a case of adenocarcinoma lower third esophagus. On radiological imaging, he also had extensive metastatic deposits in the liver, pleura, and regional and paravertebral lymph nodes.

\section{Introduction}

The condition leptomeningeal metastasis, carcinomatosis, or neoplastic meningitis involves cases where the tumor spreads from its original site to the meningeal covering. In this tumor, cells are also found circulating in the cerebrospinal fluid (CSF) ${ }^{1}$. It is extremely rare to note neoplastic meningitis in esophageal cancer. In the literature, esophageal carcinoma metastasis to the brain was found in only 36 patients in Japan. ${ }^{2}$ One of the therapies that is used in its management includes intrathecal approach of administration of chemotherapy and radiotherapy. There are no specific treatment guidelines described in the literature.

We hereby report a case of a 55-year-old male having extensive leptomeningeal carcinomatosis.

\section{Case Report}

A 55-year-old male presented with history of pain in abdomen, headache, and vomiting since the last 7 days. He was a known case of carcinoma esophagus since the last 9 months and was on treatment. PET CT F18-FDG (whole body) showed growth in the lower thoracic esophagus to GE junction, measuring $3.5 \times 3.1 \times 1 \mathrm{~cm}$ and protruding in the stomach with thickness measuring $2.5 \mathrm{~cm}$. The growth in the lower esophagus, with extension to GE junction and part of the lesser curvature of stomach was evident. Also, nodal metastasis to aortoesophageal, gastrohepatic ligament, aortocaval, and retrocaval nodes were noted. Both lobes of liver showed multiple nodules, with the largest measuring $4.6 \times 3 \times 2.5 \mathrm{~cm}$. Bilateral pleura-based nodules in the subpleural area were also suggestive of metastasis. Left iliac bone and humeral head showed lytic skeletal lesion.

Esophageal biopsy from lower one third of esophageal growth reported as moderate to poorly differentiated adenocarcinoma. Patient received 12 cycles of chemotherapy.

On 7 months follow-up, MRI of brain (plain and contrast) showed diffuse linear postcontrast enhancement along the surface of brain parenchyma to the supra- and intratentorial subarachnoid space. The finding is suggestive of diffuse leptomeningeal carcinomatosis (-Figs. 2 and $\mathbf{3}$ ). The CSF cytology analysis showed $5 \mathrm{~mL}$, slightly hazy fluid with raised protein levels. The CSF microscopy findings were RBC 2 to 3/hpf, WBC-total leucocyte count $-160 / \mathrm{mm}^{3}$ with differential leucocyte count-neutrophil 30\% and lymphocyte $70 \%$ along with many clusters of scattered neoplastic cells which are variable sized. The tumor cells showed moderate pleomorphism. The nuclei were hyperchromatic to vesicular with prominent nucleoli. The tumor cells had less cytoplasm. A few scattered tumor giant cells were noted. The findings suggested a positive result for adenocarcinoma cells in cerebrospinal fluid. ( - Fig. 1A, B) 

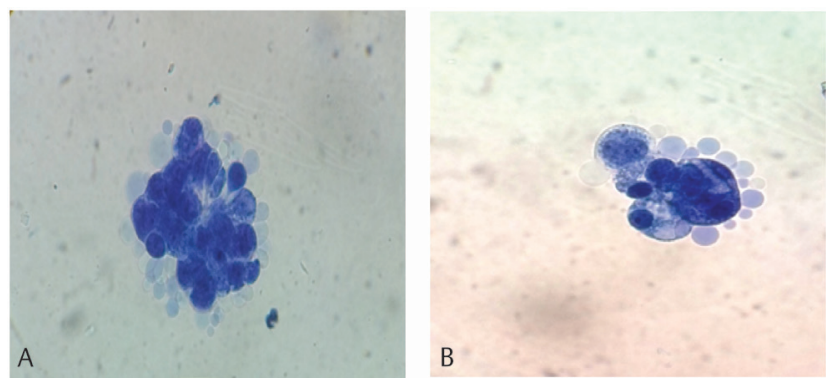

Fig. 1 (A) Cerebro spinal fluid showing red blood cell and neoplastic adenocarcinoma cells in clusters. (Leishman's stain,100x). (B) Cluster of neoplastic adenocarcinoma cells in cerebrospinal fluid (Leishman's stain, 100x).

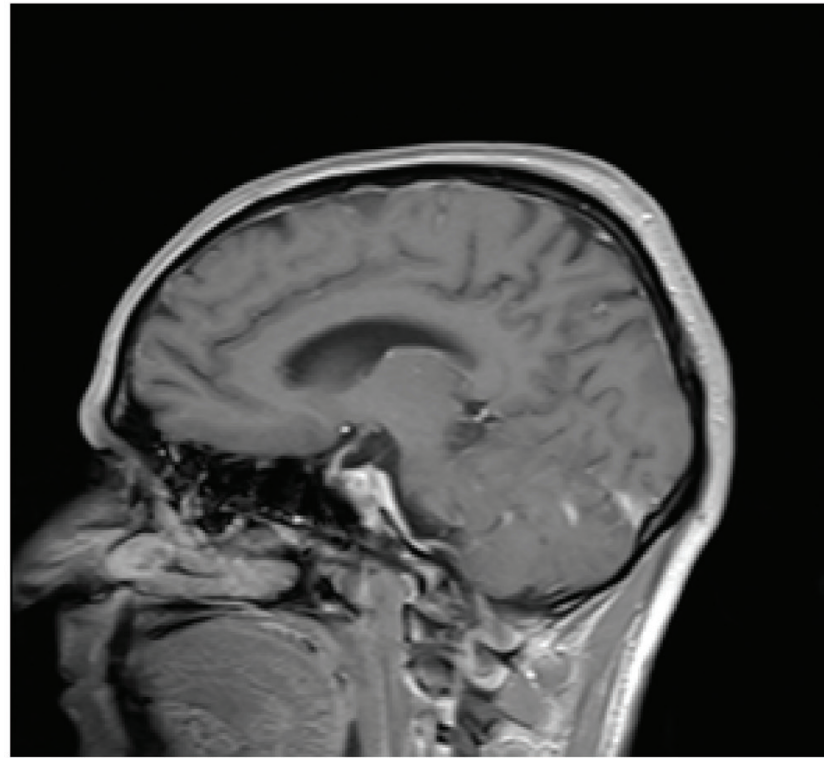

Fig. 2 Cerebrospinal fluid showing red blood cell and neoplastic adenocarcinoma cells in clusters. (Leishman's stain,100×).

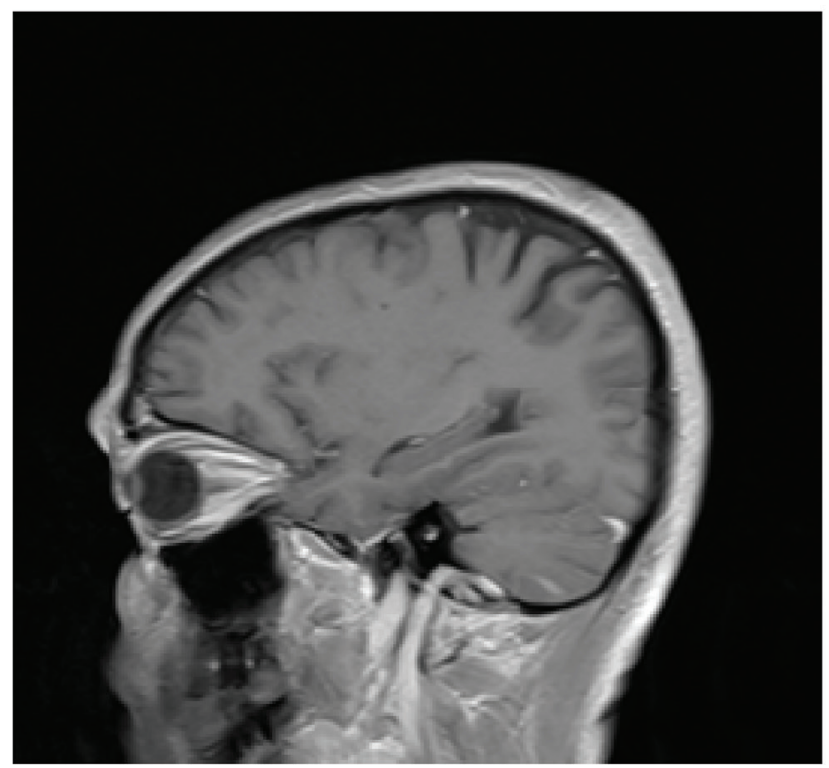

Fig. 3 MRI brain showed diffuse linear post contrast enhancement along the surface of brain parenchyma supra and intra-tentorial with in subarachanoid space.

\section{Discussion}

The esophageal cancer generally has a poor outcome. Most patients present at an advanced stage at the timing of diagnosis. These tumors have a tendency to spread via the lymphatics and hematogenous route. The common sites for metastasis are lungs, liver, peritoneum, bone, and kidney. ${ }^{2-4}$ The local continues spread to stomach and adjust structure noted commonly, while the metastasis deposited in the brain was infrequent. 5,6

It is very rarely noted that solid tumors showed carcinomatosis meningitis. It happens in 5 to $10 \%$ of patients afflicted with carcinoma lung, melanoma or breast cancer, while it is extremely rare in esophageal cancer. ${ }^{7}$ The incidence of leptomeningeal involvement in esophageal adenocarcinoma is $0.16 \%$ and gastric adenocarcinoma is $0.14 \% .^{7-9}$ Leptomeningeal carcinomatosis may present with variable manifestations like limb weakness, sensory loss, altered mental status, seizures, facial pain, visual disturbances, and radicular pain. The signs and symptoms related to CSF obstruction such as nausea, vomiting, headache, and confusion were noted. The detection of carcinomatosis meningitis secondary to esophageal adenocarcinoma is difficult to diagnose on CT and MRI of head. There was highsensitivity (91\%) for detecting in CSF examination by lumbar puncture. ${ }^{10}$ On radiological examination, it showed that the majority of metastatic tumor cells were diffuse and infiltrated into the meninges or subarachnoid space, with no significant space-occupying lesion or effect. In our care along with CT, MRI findings, we were able to diagnose this case in the CSF cytology, which showed neoplastic cells of metastatic adenocarcinoma.

The gastroesophageal adenocarcinoma is the most difficult malignancies to treat, with poor prognosis and overall survival range from 4 to 16 weeks after diagnosis. ${ }^{8}$ The various treatment practices are as follows: surgical excision, radiation, and chemotherapy. For symptoms of pain, headache treated by corticosteroids. Currently, there is no effective treatment for leptomeningeal carcinoma. Palliative care for neurodeficits, hydrocephalous, encephalopathy and systemic involvement are essential aspects of treatment. The overall outcome of leptomeningeal carcinomatosis remains poor. The rate of detection is increasing with incorporation and utilization of CSF examination, CSF tumor markers, newer radiological imaging techniques, etc., to detect tumorspecific somatic mutations.

\section{Conclusion}

We report leptomeningeal carcinomatosis in a case of moderately differentiated adenocarcinoma in esophagus for its clinical, radiological, and cytological findings.

\section{Conflict of Interest}

None. 


\section{References}

1 Mittica G, Senetta R, Richiardi L, et al. Meningeal carcinomatosis underdiagnosis and overestimation: incidence in a large consecutive and unselected population of breast cancer patients. BMC Cancer 2015;15:1021

2 Ogawa K, Toita T, Sueyama H, et al. Brain metastases from esophageal carcinoma: natural history, prognostic factors, and outcome. Cancer 2002;94(3):759-764

3 Mandard AM, Chasle J, Marnay J, et al. Autopsy findings in 111 cases of esophageal cancer. Cancer 1981;48(2):329-335

4 Roth J, Putnam J, Rich T, Forastiere A, Cancer of the esophagus. In: DeVita V Jr, Hellman S, Rosenberg S, eds. Cancer. Principles and Practice of Oncology. Vol 2. New-York: Lippincott-Raven, 1997 980-1021

5 Gabrielsen TO, Eldevik OP, Orringer MB, Marshall BL. Esophageal carcinoma metastatic to the brain: clinical value and costeffectiveness of routine enhanced head CT before esophagectomy. AJNR Am J Neuroradiol 1995;16(9):1915-1921
6 Takeshima H, Kuratsu J, Nishi T, et al. Metastatic brain tumours from esophageal carcinoma: neuro-imaging and clinicopathological characteristics in Japanese patients. Acta Neurochir (Wien) 2001;143(1):31-35, discussion 35-36

7 Giglio P, Weinberg JS, Forman AD, Wolff R, Groves MD. Neoplastic meningitis in patients with adenocarcinoma of the gastrointestinal tract. Cancer 2005;103(11):2355-2362

8 Oh S, Kim S, et al. Leptomeningeal carcino-matosis as the first manifestation of gastric cancer: multicentre retrospective analysis of 54 cases. J Clin Oncol 2009;15(40):5086-5090

9 Rizvi S, Fateh S, Abbi KK, Jiang Y. Leptomeningeal carcinomatosis as the only manifestation of disease in recurrent gastresophageal cancers. Gastrointest Cancer Res 2011;4(2):68-71

10 Wasserstrom WR, Glass JP, Posner JB. Diagnosis and treatment of leptomeningeal metastases from solid tumors: experience with 90 patients. Cancer 1982;49(4):759-772 